\title{
The Short ITS2 Sequence Serves as an Efficient Taxonomic Sequence Tag in Comparison with the Full-Length ITS
}

\author{
Jianping Han, ${ }^{1}$ Yingjie Zhu, ${ }^{1}$ Xiaochen Chen, ${ }^{1}$ Baoshen Liao, ${ }^{1}$ Hui Yao, ${ }^{1}$ \\ Jingyuan Song, ${ }^{1}$ Shilin Chen, ${ }^{1}$ and Fanyun Meng ${ }^{2}$ \\ ${ }^{1}$ Institute of Medicinal Plant Development, Chinese Academy of Medical Sciences and Peking Union Medical College, \\ Beijing 100094, China \\ ${ }^{2}$ College of Resources Science \& Technology, Beijing Normal University, Beijing 100875, China
}

Correspondence should be addressed to Shilin Chen; slchen@implad.ac.cn and Fanyun Meng; mfy@bnu.edu.cn

Received 31 October 2012; Accepted 26 December 2012

Academic Editor: Abdurrahman Saydut

Copyright (C) 2013 Jianping Han et al. This is an open access article distributed under the Creative Commons Attribution License, which permits unrestricted use, distribution, and reproduction in any medium, provided the original work is properly cited.

\begin{abstract}
An ideal DNA barcoding region should be short enough to be amplified from degraded DNA. In this paper, we discuss the possibility of using a short nuclear DNA sequence as a barcode to identify a wide range of medicinal plant species. First, the PCR and sequencing success rates of ITS and ITS2 were evaluated based entirely on materials from dry medicinal product and herbarium voucher specimens, including some samples collected back to 90 years ago. The results showed that ITS2 could recover $91 \%$ while ITS could recover only $23 \%$ efficiency of PCR and sequencing by using one pair of primer. Second, 12861 ITS and ITS2 plant sequences were used to compare the identification efficiency of the two regions. Four identification criteria (BLAST, inter- and intradivergence Wilcoxon signed rank tests, and TaxonDNA) were evaluated. Our results supported the hypothesis that ITS2 can be used as a minibarcode to effectively identify species in a wide variety of specimens and medicinal materials.
\end{abstract}

\section{Introduction}

1.1. DNA Barcoding of Degraded DNA Materials. DNA barcoding takes advantage of short standard sequences to discover and identify species [1]. An ideal DNA barcode should be short enough to be amplified from archival specimens using universal primers. The term "minimalist barcode" was first defined by Herbert as a tool to overcome the low PCR efficiency of cytochrome c-oxidase subunit 1 (CO1) in archival animal specimens in museums, and the possibility of identifying animal specimens using a region of approximately $200 \mathrm{bp}$ was discussed. The results of that study showed that minibarcodes can be isolated from different types of specimens, including museum samples, trace tissue samples with degraded DNA and other specimens, from which the acquisition of a full-length barcode (CO1) is not feasible [2]. The amplification of DNA from herbarium specimens is also important for barcoding studies because it is often necessary to confirm the species identification of fresh specimens by comparing their sequences with those of older museum specimens [3]. Additionally, most of the medicinal materials available in the market are dry and have been stored for long periods; thus, it is very difficult to amplify long DNA regions from some of these materials, which prevent the use of DNA barcodes for herb identification.

1.2. The Trend of Core Plant DNA Barcodes. The Plant Working Group of the Consortium for the Barcode of Life (CBOL) recommended the use of a combination of matk and $r b c L$ as a barcode for land plants [4], and internal transcribed spacer (ITS)/internal transcribed spacer 2 (ITS2) was proposed as a supplemental marker for further study. The ITS sequence contains enough variable sites for species identification in many samples [5-9], but ITS could not be amplified from approximately $12 \%$ of herbarium samples [3], because ITS1 is too variable to guarantee reliable alignments and contains variable indels (insertions/deletions) at this taxonomic level. Additionally, multiple functional copies exist in many taxa. Thus, ITS was excluded as a universal 
land plant barcode in the earlier stages. In contrast, ITS2 is considered to have evolved in concert, which leads to a homogenization of all the copies of this gene throughout the genome and in most organisms ITS2 was treated as a single locus. Thus, the ITS2 region might be a suitable marker for taxonomic classification [10-12]. Recently, ITS2 has been suggested as a useful barcode for medicinal plants [13-17], as a universal DNA barcode to identify plants and as a complementary locus of CO1 to identify animals [18]. The China Plant Barcode of Life Group considered ITS2 to be a useful alternative to ITS because it is more easily amplified and sequenced [19]. In addition, the secondary structure of ITS2 was shown to be an efficient tool for biological species identification [20, 21].

Here, we demonstrated the effectiveness of ITS2 as a minibarcode in comparison with the full-length ITS for the identification of a wide range of archived plant species. An initial set of 100 medicinal samples from museum specimens and the herb market was tested to determine the PCR and sequencing efficiencies of ITS and ITS2. A second set of 12861 sequences, representing 8313 species collected from GenBank, was examined to compare the identification abilities of ITS and ITS2. This work aims to provide an evaluation of ITS2 as a minibarcode for large samples.

\section{Materials and Methods}

2.1. Plant Material. The initial set of 100 museum medicinal specimens and herbal products from 92 species representing 5 orders (see Table 1 of the Supplementary Material available online at http://dx.doi.org/10.1155/2013/741476) was collected from the Buozhou herbal market and from specimens at the Institute of Medicinal Plant Development, some of which were collected 90 years ago, to test the efficiency of PCR and sequencing. All the samples were authenticated at the species level by Professor Yulin Lin (Institute of Medicinal Plant Development, Chinese Academy of Medical Sciences). A second set of sequences for the identification efficiency analysis presented in this paper was obtained from the GenBank nucleotide sequence database. We carried out a bioinformatics analysis using all ITS sequences present in GenBank matching the search pattern " $18 \mathrm{~S}$ ribosomal RNA gene; internal transcribed spacer 1, 5.8S ribosomal RNA gene, and internal transcribed spacer 2, and $28 \mathrm{~S}$ ribosomal RNA gene." Partial sequences, fungal sequences, and sequences of less than $100 \mathrm{bp}$ were removed. A flowchart is shown in Figure 1. The complete ITS2 and full-length ITS regions were annotated using the Hidden Markov Model (HMM) [22] and ITS plant model, respectively, which rely on highly similar and correctly annotated reference sequences present in the public database. Ultimately, 12861 sequences representing 8313 species from 1699 genera were obtained (GenBank accession numbers are listed in Table 2S) and used to analyze the identification efficiencies of ITS and ITS2.

2.2. DNA Extraction, PCR Amplification and Sequencing. Total genomic DNA was extracted from specimens using the Plant Genomic DNA Kit (Tiangen Biotech Beijing Co., Ltd., China) according to the manufacturer's instructions. The primer sequences for ITS2 were described by Chen et al. [13]. ITS was amplified using the primers ITS5 and ITS4 [23]. The PCR conditions and sequences used to amplify the two regions (ITS and ITS2) were based on the methods described by Kress et al. and Chen et al. [1, 13, 24, 25].

2.3. Analysis Method. Six parameters were used to characterize the interspecific and intraspecific divergences, according to a previously described method [13]. Three of the parameters were used to estimate the interspecific variability: average inter-specific distance, average theta prime, and smallest inter-specific distance. The other three parameters were used to evaluate the intraspecific divergence: average intraspecific difference, theta, and average coalescent depth. The Wilcoxon signed rank test was used as described previously [13, 26, 27]. Basic Local Alignment Search Tool (BLAST1) was performed to identify the species [13]. The TaxonDNA software was used to calculate the identification efficiency [28, 29].

\section{Results}

3.1. PCR and Universal Primers. To evaluate the efficiency of PCR and sequencing, 100 medicinal samples from herbal market and museum specimens, including 91 species from 5 orders, were tested; $16 \%$ of the samples were obtained from the herb market, and the remaining $84 \%$ were obtained from the Institute of Medicinal Plant Development. The ITS primer pair yielded a recovery rate of only $23 \%$, compared with the $91 \%$ recovery rate for ITS2. All sequences were submitted to GenBank (the GenBank accession numbers are listed in Table 1S, Supplementary Material). The small size of ITS2 facilitates its amplification by universal primers, even in samples with partially degraded DNA.

\subsection{Species Identification}

3.2.1. Comparison of Inter- and Intraspecific Divergences. Comparison of the inter- and intraspecies sequence variation was an important aspect of the barcoding identification. For the 12861 ITS and ITS2 sequences, which contained 8313 species from 1699 genera, the average lengths of ITS and ITS2 were $634 \mathrm{bp}$ and $233 \mathrm{bp}$, respectively. The comparison of the inter- and intraspecific genetic distances revealed that the ITS2 region exhibited a higher inter-specific divergence according to the three inter-specific parameters (Table 1). Another advantage of ITS2 is that its conserved secondary structure is associated with relatively low intra-specific variation. The combination of a conserved secondary structure with a variable sequence appears to be a major benefit of using ITS2 [30].

The differences in the percent sequence divergence between loci were tested using the Wilcoxon signed rank test. The results showed that ITS2 was a more variable barcode (Table 2). ITS contained a conserved 5.8S region, which decreased the comparative divergence. Based on these results, ITS2 demonstrates sufficient variation to differentiate plants. 


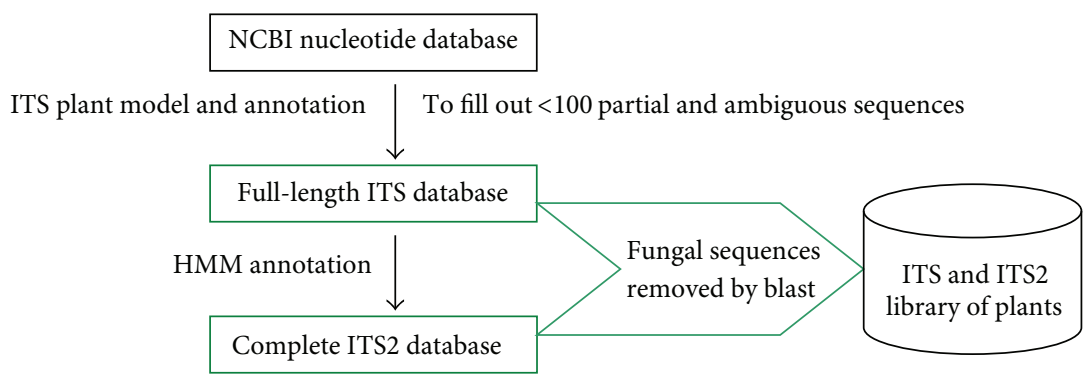

FIgURE 1: Flowchart of data analysis.

TABLE 1: Analysis of interspecific divergence and intraspecific variation of candidate barcodes.

\begin{tabular}{lcr}
\hline Marker & ITS & ITS2 \\
\hline Avg_intra_avg & $0.0145 \pm 0.0467$ & $0.0188 \pm 0.0792$ \\
Avg_intra_max & $0.0203 \pm 0.0701$ & $0.0327 \pm 0.2589$ \\
Avg_intra_between_intra-species & $0.0308 \pm 0.1182$ & $0.0533 \pm 0.3202$ \\
Avg_interbyG_avg & $0.0736 \pm 0.0688$ & $0.0959 \pm 0.1047$ \\
Avg_interbyG_min & $0.0329 \pm 0.0517$ & $0.0402 \pm 0.0719$ \\
Avg_between_interbyGenus & $0.0752 \pm 0.0620$ & $0.1000 \pm 0.1138$ \\
\hline
\end{tabular}

3.2.2. BLAST-Based Identification. BLAST1 was used to evaluate the efficiencies of ITS2 and ITS. ITS and ITS2 successfully identified $89.2 \%$ and $79.2 \%$ of specimens, respectively, at the species level and $97.5 \%$ and $93.8 \%$, respectively, at the genus level (Table 3). Additionally, the significantly smaller size of ITS2 (average length of approximately $233 \mathrm{bp}$ ) compared with that of ITS (average length of approximately $634 \mathrm{bp}$ ) makes ITS2 a better candidate for barcoding studies.

To estimate the respective identification efficiency per genus, genera that contain at least 20 species were selected independently (Table 4). In $85 \%(68 / 80)$ of the genera, the success rates of ITS and ITS2 are identical. ITS had an identification efficiency superior to that of ITS2 in the following 12 genera: Gunnera, Luzula, Strobilanthes, Nepeta, Dionysia, Adenia, Clidemia, Sedum, Indigofera, Kalanchoe, Pilea, and Melampodium. Of the 603 genera that contain at least 3 samples, ITS2 and ITS had the same identification efficiency in 394 genera (65.3\%), and ITS and ITS2 shared a $100 \%$ identification efficiency at the species level in 345 genera (57.2\%) (Table 3S).

3.2.3. TaxonDNA Identification. We also used TaxonDNA to assess the accuracy of species identification based on ITS and ITS2. TaxonDNA is an alignment-based parametric clustering program that determines the closest match of a sequence by comparing it with all other sequences in the aligned data set. If the compared sequences were from the same species, the identification was considered successful, whereas mismatched names were counted as failures. Cases with several equally good best matches from different species were considered ambiguous [29]. In this study, the successful identification rates of the "best match" were $67.88 \%$ and $60 \%$ for ITS and ITS2, respectively. The ambiguous identification rates of ITS and ITS2 were $14.9 \%$ and $0 \%$, respectively, and the misidentification rates were $17.2 \%$ and $40 \%$, respectively. The dataset contained 8607 sequences with duplication.

We used TaxonDNA to set the threshold value. All sequences without a match below the $97 \%$ threshold value remained unidentified. If the compared sample names were identical, the identification was considered correct; if the sequence names were mismatched, the identification was considered a failure. When several equally good best matches that belonged to a minimum of two species were found, the identification was considered ambiguous [29, 31]. The successful identification rates under the "best close match" were $62.53 \%$ and $32 \%$ for ITS and ITS2, respectively. The ambiguous identification rates of ITS and ITS2 were $14.0 \%$ and $0 \%$, respectively. The misidentification rates of ITS and ITS2 were $7.28 \%$ and $0 \%$, respectively. The remaining samples were considered unidentified because they had no matches below the threshold value. The nonmatch ratios of ITS and ITS2 were $16.2 \%$ and $68 \%$, respectively (Table 5). ITS provided slightly superior successful identification and misidentification rates compared with ITS2, but ITS2 provided a lower ambiguous identification rate $(0 \%$ versus $14.9 \%$ and $14.0 \%$ under the "best match" and "best close match," resp., for ITS).

\section{Discussion}

4.1. PCR and Sequencing Success Rates. Many museum specimens are very useful for DNA barcoding studies. However, high-quality DNA can be difficult to obtain from these specimens, making PCR amplification and sequencing inefficient. In this study, we recovered short ITS2 sequences from more than $90 \%$ of the herbal specimens representing 5 orders, whereas the recovery rate for ITS with a single primer set was only $23 \%$. This discrepancy between the two regions arises because ITS is very long relative to ITS2, and ITS require a 
TABLE 2: Wilcoxon signed rank tests of inter- and intraspecific divergences among loci.

\begin{tabular}{lcc}
\hline Divergence & Interrelative ranks, $n, P$ value & Result \\
\hline Interspecific & $W+=2.92 E 8, W-=1.14471579 E 8, n=28495, P=0$ & ITS2 $>$ ITS \\
Intraspecific & $W+=18423293.50, W-=11658352.50, n=7756, P=6.026542085865164 E-66$ & ITS2 $>$ ITS \\
\hline
\end{tabular}

TABLE 3: Identification efficiency of ITS and ITS2 by using BLAST.

\begin{tabular}{lcccccc}
\hline Marker & Samples & Genus & Species & Length & Identification success at genus level & Identification success at the species level \\
\hline ITS & 12861 & 1699 & 8313 & 633.7 & $97.5 \%$ & $89.2 \%$ \\
ITS2 & 12861 & 1699 & 8313 & 232.6 & $93.8 \%$ & $79.2 \%$ \\
\hline
\end{tabular}

variety PCR conditions and additives for successful amplification [32]. Another potential explanation is that intact DNA was difficult to extract from these samples due to the degradation that occurred in the museum specimens during the long storage period and in the herbs from the market during harvesting, processing, and storage. In contrast, the ITS2 region can be easily amplified and sequenced with conserved primers. Due to its relatively short length, the ITS2 minibarcode could be amplified with greater success than the full-length ITS sequences in almost all groups.

4.2. Identification Efficiency of ITS and ITS2. To determine whether barcode gaps are present in this study, the relationships between the inter- and intraspecific divergences were compared for each species. For the 12861 samples, ITS and ITS2 could identify $97.5 \%$ and $93.8 \%$ of genera, respectively, by the BLAST method. The full-length ITS could identify approximately $89.2 \%$ of the species, and the mini-DNA barcode ITS2 successfully identified approximately $79.2 \%$ of the species, which is higher than the CBOL proposed plant combination of matK and $r b c L(70 \%)[4,5]$.

TaxonDNA was also used to compare the identification efficiencies of ITS and ITS2, and the result appeared to be similar to that obtained by the BLAST method. ITS had slightly superior successful identification and misidentification rates compared with ITS2, but the ambiguous identification rate of ITS2 was $0 \%$, whereas that of ITS was $14.9 \%$ and $14.0 \%$ under the "best match" and "best close match" algorithms, respectively. The zero ambiguous identification rate of ITS2 may be due to its conserved secondary structure. The secondary structure of ITS2 has proven useful for diagnostic purposes at the species level [21], which might reduce the ambiguous identification rates and increase the correctness of the barcoding analysis. Evidence has shown that a combination of nucleotide and secondary structure data can overcome some of the limitations of ITS2 [33] and that the ITS2 sequence and secondary structure (sequence-structure) provided the most accurate results, which benefit from the secondary structure [30, 34]. Thus, the use of the ITS2 secondary structures would be extremely helpful to address the challenges of species identification and classification.

4.3. ITS2 versus ITS: Advantages and Limitations. ITS2 has many advantages that make it superior to ITS. First, it is important that species be defined correctly for DNA barcoding by systematic analysis [3]. ITS2 regions with secondary structures are more conserved than the DNA sequences alone, which could provide information that is useful for the cladistic inference of relationships [35], and the ITS2 sequence-structure information provides a compensatory base changes (CBCs) analysis result that correlates with the biological species concept [21]. Thus, ITS2 has been considered a double-edged tool for evolutionary comparisons in eukaryotes [12].

Second, millions of species will need to be sequenced for a global barcode project, and this would be extremely costly using standard sequencing methods. The read lengths provided by high-throughput sequencing would be sufficient to build a database of ITS2 mini-DNA barcode sequences. High-throughput sequencing technology uses an emulsion PCR approach to simultaneously amplify several thousand 100-200 bp DNA molecules in one reaction and yields a large number of short sequences with a lower cost than standard approaches. Mello proved that the ITS2 read length obtained by high-throughput 454 sequencing provided adequate information for taxon assignment [36]. Song et al. used high-throughout 454 sequencing to successfully obtain a large number of ITS2 sequences in one reaction [37]. The amenability to high-throughput approaches and high identification efficiency makes the ITS2 minibarcode useful for projects involving a large number of environmental samples.

Third, although ITS2 was less powerful than ITS for resolving some closely related species, it showed many advantages, especially in identifying herbs and specimens containing degraded DNA. ITS2 sequences could be used to design taxon-specific probes for the rapid identification of plants [38], and an ITS2 microarray has been used to successfully separate species with sequence identities up to $97 \%$ [39]. Considering the short length and high identification efficiency of the ITS2 sequence, we confirmed that this very short barcode sequence is valuable for the identification of old specimens and medicinal materials.

Finally, there are hundreds of copies of ITS within a genome. Nonetheless, ITS2 can be considered a single locus in the whole genome of most organisms [10, 12, 37], including Panax ginseng and Panax quinquefolius (unpublished), making ITS2 more suitable as a barcode than ITS.

This study demonstrated the potential of the ITS2 minibarcode for DNA barcoding analyses. ITS2 showed high 
TABLE 4: Comparing of the identification rates of ITS with ITS2 in genera with more than 20 species.

\begin{tabular}{|c|c|c|c|c|c|c|c|c|c|}
\hline \multirow{3}{*}{ Genus } & \multirow{3}{*}{ Species } & \multirow{3}{*}{ Samples } & \multicolumn{2}{|c|}{ Unidentified species } & \multirow{3}{*}{ Genus } & \multirow{3}{*}{ Species } & \multirow{3}{*}{ Samples } & \multicolumn{2}{|c|}{ Unidentified species } \\
\hline & & & & & & & & & \\
\hline & & & ITS & ITS2 & & & & ITS & ITS2 \\
\hline Acer & 57 & 341 & $0.30 \%$ & $0.30 \%$ & Maxillaria & 245 & 506 & $0.20 \%$ & $0.20 \%$ \\
\hline Adenia & 37 & 39 & $0.00 \%$ & $2.60 \%$ & Melampodium & 39 & 93 & $0.00 \%$ & $1.10 \%$ \\
\hline Begonia & 42 & 71 & $1.40 \%$ & $1.40 \%$ & Miconia & 166 & 175 & $0.60 \%$ & $0.60 \%$ \\
\hline Calceolaria & 64 & 64 & $1.60 \%$ & $1.60 \%$ & Morinda & 20 & 24 & $0.00 \%$ & $0.00 \%$ \\
\hline Caragana & 28 & 39 & $2.60 \%$ & $2.60 \%$ & Muraltia & 67 & 67 & $1.50 \%$ & $1.50 \%$ \\
\hline Carex & 73 & 80 & $1.30 \%$ & $1.30 \%$ & Nepeta & 31 & 31 & $0.00 \%$ & $3.20 \%$ \\
\hline Castilleja & 21 & 21 & $4.80 \%$ & $4.80 \%$ & Nicotiana & 31 & 43 & $2.30 \%$ & $2.30 \%$ \\
\hline Cineraria & 20 & 23 & $4.30 \%$ & $4.30 \%$ & Oncidium & 76 & 92 & $1.10 \%$ & $1.10 \%$ \\
\hline Clidemia & 39 & 40 & $0.00 \%$ & $2.50 \%$ & Oxalis & 168 & 185 & $0.50 \%$ & $0.50 \%$ \\
\hline Cliffortia & 30 & 33 & $3.00 \%$ & $3.00 \%$ & Panicum & 29 & 39 & $2.60 \%$ & $2.60 \%$ \\
\hline Clusia & 26 & 63 & $1.60 \%$ & $1.60 \%$ & Paphiopedilum & 39 & 55 & $1.80 \%$ & $1.80 \%$ \\
\hline Coffea & 79 & 106 & $0.90 \%$ & $0.90 \%$ & Pentas & 26 & 27 & $3.70 \%$ & $3.70 \%$ \\
\hline Costus & 45 & 75 & $1.30 \%$ & $1.30 \%$ & Phyllanthus & 64 & 82 & $1.20 \%$ & $1.20 \%$ \\
\hline Crinum & 27 & 27 & $3.70 \%$ & $3.70 \%$ & Pilea & 63 & 66 & $0.00 \%$ & $1.50 \%$ \\
\hline Croton & 45 & 45 & $2.20 \%$ & $2.20 \%$ & Planchonella & 37 & 42 & $2.40 \%$ & $2.40 \%$ \\
\hline Cuscuta & 44 & 69 & $1.40 \%$ & $1.40 \%$ & Plantago & 68 & 88 & $1.10 \%$ & $1.10 \%$ \\
\hline Cyrtochilum & 32 & 42 & $2.40 \%$ & $2.40 \%$ & Poa & 44 & 56 & $1.80 \%$ & $1.80 \%$ \\
\hline Dichaea & 34 & 58 & $1.70 \%$ & $1.70 \%$ & Potentilla & 31 & 32 & $3.10 \%$ & $3.10 \%$ \\
\hline Dionysia & 35 & 37 & $0.00 \%$ & $2.70 \%$ & Pouteria & 24 & 27 & $0.00 \%$ & $0.00 \%$ \\
\hline Disterigma & 20 & 24 & $4.20 \%$ & $4.20 \%$ & Rhodiola & 20 & 47 & $2.10 \%$ & $2.10 \%$ \\
\hline Draba & 122 & 273 & $0.40 \%$ & $0.40 \%$ & Rhus & 21 & 28 & $3.60 \%$ & $3.60 \%$ \\
\hline Elymus & 35 & 98 & $1.00 \%$ & $1.00 \%$ & Ruellia & 98 & 116 & $0.90 \%$ & $0.90 \%$ \\
\hline Epimedium & 20 & 23 & $4.30 \%$ & $4.30 \%$ & Salix & 25 & 33 & $3.00 \%$ & $3.00 \%$ \\
\hline Ficus & 147 & 152 & $0.70 \%$ & $0.70 \%$ & Salvia & 62 & 124 & $0.80 \%$ & $0.80 \%$ \\
\hline Fritillaria & 38 & 38 & $2.60 \%$ & $2.60 \%$ & Sauropus & 31 & 37 & $0.00 \%$ & $0.00 \%$ \\
\hline Gagea & 72 & 141 & $0.70 \%$ & $0.70 \%$ & Scaevola & 42 & 48 & $2.10 \%$ & $2.10 \%$ \\
\hline Garcinia & 43 & 92 & $1.10 \%$ & $1.10 \%$ & Scaphyglottis & 34 & 41 & $0.00 \%$ & $0.00 \%$ \\
\hline Gomesa & 44 & 51 & $2.00 \%$ & $2.00 \%$ & Sedum & 54 & 56 & $0.00 \%$ & $1.80 \%$ \\
\hline Gunnera & 20 & 20 & $0.00 \%$ & $5.00 \%$ & Selago & 21 & 22 & $4.50 \%$ & $4.50 \%$ \\
\hline Hoya & 32 & 81 & $1.20 \%$ & $1.20 \%$ & Senecio & 76 & 89 & $1.10 \%$ & $1.10 \%$ \\
\hline Indigofera & 52 & 63 & $0.00 \%$ & $1.60 \%$ & Sideroxylon & 42 & 42 & $2.40 \%$ & $2.40 \%$ \\
\hline Kalanchoe & 49 & 67 & $0.00 \%$ & $1.50 \%$ & Silene & 20 & 28 & $3.60 \%$ & $3.60 \%$ \\
\hline Kniphofia & 51 & 96 & $1.00 \%$ & $1.00 \%$ & Stevia & 71 & 91 & $1.10 \%$ & $1.10 \%$ \\
\hline Leandra & 26 & 26 & $3.80 \%$ & $3.80 \%$ & Strobilanthes & 29 & 29 & $0.00 \%$ & $3.40 \%$ \\
\hline Leontodon & 22 & 31 & $3.20 \%$ & $3.20 \%$ & Swartzia & 22 & 30 & $0.00 \%$ & $0.00 \%$ \\
\hline Luzula & 25 & 29 & $0.00 \%$ & $3.40 \%$ & Taraxacum & 27 & 215 & $0.50 \%$ & $0.50 \%$ \\
\hline Macaranga & 38 & 38 & $2.60 \%$ & $2.60 \%$ & Tiquilia & 27 & 303 & $0.30 \%$ & $0.30 \%$ \\
\hline Macrocarpaea & 46 & 81 & $1.20 \%$ & $1.20 \%$ & Tolumnia & 21 & 23 & $4.30 \%$ & $4.30 \%$ \\
\hline Mallotus & 29 & 33 & $0.00 \%$ & $0.00 \%$ & Trifolium & 222 & 257 & $0.40 \%$ & $0.40 \%$ \\
\hline Masdevallia & 34 & 34 & $2.90 \%$ & $2.90 \%$ & Veronica & 132 & 153 & $0.70 \%$ & $0.70 \%$ \\
\hline
\end{tabular}

TABLE 5: Identification success based on "best match" and "best close match."

\begin{tabular}{lcccc}
\hline & & Best match & \multicolumn{2}{c}{ Best close match } \\
& ITS & ITS2 & ITS & 32.53 \\
Correct identification (\%) & 67.88 & 60 & 14.0 & 00 \\
Ambiguous identification (\%) & 15 & 0 & 7.28 & 0.00 \\
Incorrect identification (\%) & 17 & 40 & 16.20 & 0.00 \\
Without any match closer than 3.0\% (\%) & - & - & 68.00 \\
\hline
\end{tabular}


sequence variability among 12861 samples from 8313 species. An ideal DNA barcoding marker for taxonomic classification should be fast-evolving to allow classification at the species level but must also contain highly conserved priming sites and be highly reliable for DNA amplification and sequencing [40]. The ITS2 region meets the expected criteria of a global DNA barcode. Our analysis supports the use of the ITS2 minibarcode as a "universal DNA barcode" for the rapid identification of medicinal materials and specimens.

\section{Conflict of Interests}

The authors declare that no conflict of interests exists in this paper.

\section{Acknowledgments}

This work was supported by the National Natural Science Foundation of China (Grant nos. 81001608 and 81130069). The authors thank their colleagues who helped in the sample collection, identification, laboratory work, and paper preparation, including Professor Yulin Lin, Chang Liu, and many others.

\section{References}

[1] W. J. Kress, K. J. Wurdack, E. A. Zimmer, L. A. Weigt, and D. H. Janzen, "Use of DNA barcodes to identify flowering plants," Proceedings of the National Academy of Sciences of the United States of America, vol. 102, no. 23, pp. 8369-8374, 2005.

[2] M. Hajibabaei, M. A. Smith, D. H. Janzen, J. J. Rodriguez, J. B. Whitfield, and P. D. N. Hebert, "A minimalist barcode can identify a specimen whose DNA is degraded," Molecular Ecology Notes, vol. 6, no. 4, pp. 959-964, 2006.

[3] D. Rubinoff, S. Cameron, and K. Will, "Are plant DNA barcodes a search for the Holy Grail?" Trends in Ecology and Evolution, vol. 21, no. 1, pp. 1-2, 2006.

[4] C.P.W. Group, "A DNA barcode for land plants," Proceedings of the National Academy of Sciences of the United States of America, vol. 106, no. 31, pp. 12794-12797, 2009.

[5] H. Yousefzadeh, A. H. Colagar, M. Tabari, A. Sattarian, and M. Assadi, "Utility of ITS region sequence and structure for molecular identification of Tilia species from Hyrcanian forests, Iran," Plant Systematics and Evolution, vol. 298, no. 5, pp. 947-961, 2012.

[6] I. Álvarez and J. F. Wendel, "Ribosomal ITS sequences and plant phylogenetic inference," Molecular Phylogenetics and Evolution, vol. 29, no. 3, pp. 417-434, 2003.

[7] M. E. Mort, J. K. Archibald, C. P. Randle et al., "Inferring phylogeny at low taxonomic levels: utility of rapidly evolving cpDNA and nuclear ITS loci," American Journal of Botany, vol. 94, no. 2, pp. 173-183, 2007.

[8] B. G. Baldwin, M. J. Sanderson, J. M. Porter, M. F. Wojciechowski, C. S. Campbell, and M. J. Donoghue, "The ITS region of nuclear ribosomal DNA: a valuable source of evidence on angiosperm phylogeny," Annals of the Missouri Botanical Garden, vol. 82, no. 2, pp. 247-277, 1995.

[9] A. Quijada, A. Liston, P. Delgado, A. Vázquez-Lobo, and E. R. Alvarez-Buylla, "Variation in the nuclear ribosomal DNA internal transcribed spacer (ITS) region of pinus rzedowskii revealed by PCR-RFLP," Theoretical and Applied Genetics, vol. 96, no. 3-4, pp. 539-544, 1998.

[10] A. W. Coleman, "Pan-eukaryote ITS2 homologies revealed by RNA secondary structure," Nucleic Acids Research, vol. 35, no. 10, pp. 3322-3329, 2007.

[11] J. Schultz, S. Maisel, D. Gerlach, T. Müller, and M. Wolf, "A common core of secondary structure of the internal transcribed spacer 2 (ITS2) throughout the Eukaryota," RNA, vol. 11, no. 4, pp. 361-364, 2005.

[12] A. W. Coleman, "ITS2 is a double-edged tool for eukaryote evolutionary comparisons," Trends in Genetics, vol. 19, no. 7, pp. 370-375, 2003.

[13] S. Chen, H. Yao, J. Han et al., "Validation of the ITS2 region as a novel DNA barcode for identifying medicinal plant species," PLoS ONE, vol. 5, no. 1, Article ID e8613, 2010.

[14] T. Gao, H. Yao, J. Song et al., "Identification of medicinal plants in the family Fabaceae using a potential DNA barcode ITS2," Journal of Ethnopharmacology, vol. 130, no. 1, pp. 116-121, 2010.

[15] X. Pang, J. Song, Y. Zhu, C. Xie, and S. Chen, "Using DNA barcoding to identify species within euphorbiaceae," Planta Medica, vol. 76, no. 15, pp. 1784-1786, 2010.

[16] X. Pang, J. Song, Y. Zhu, H. Xu, L. Huang, and S. Chen, "Applying plant DNA barcodes for Rosaceae species identification," Cladistics, vol. 27, no. 2, pp. 165-170, 2011.

[17] J. P. Han, L. C. Shi, X. C. Chen, and Y. L. Lin, "Comparison of four DNA barcodes in identifying certain medicinal plants of Lamiaceae," Journal of Systematics and Evolution, vol. 50, no. 3, pp. 227-234, 2012.

[18] H. Yao, J. Song, C. Liu et al., "Use of ITS2 region as the universal DNA barcode for plants and animals," PLoS ONE, vol. 5, no. 10, Article ID e13102, 2010.

[19] D. Z. Li, L. M. Gao, H. T. Li et al., "Comparative analysis of a large dataset indicates that internal transcribed spacer (ITS) should be incorporated into the core barcode for seed plants," Proceedings of the National Academy of Sciences of the United States of America, vol. 108, no. 49, pp. 19641-19646, 2011.

[20] B. Merget, C. Koetschan, T. Hackl et al., "The ITS2 database," Journal of Visualized Experiments, vol. 61 article e3806, 2012.

[21] T. Müller, N. Philippi, T. Dandekar, J. Schultz, and M. Wolf, "Distinguishing species," RNA, vol. 13, no. 9, pp. 1469-1472, 2007.

[22] A. Keller, T. Schleicher, J. Schultz, T. Müller, T. Dandekar, and M. Wolf, "5.8S-28S rRNA interaction and HMM-based ITS2 annotation," Gene, vol. 430, no. 1-2, pp. 50-57, 2009.

[23] T. J. White, "Amplification and direct sequencing of fungal ribosomal RNA genes for phylogenetics," in PCR Protocols: A Guide to Methods and Applications, pp. 315-322, Academic Press, New York, NY, USA, 1990.

[24] C. Sass, D. P. Little, D. W. Stevenson, and C. D. Specht, "DNA barcoding in the Cycadales: testing the potential of proposed barcoding markers for species identification of Cycads," PLoS ONE, vol. 2, no. 11, Article ID e1154, 2007.

[25] S. J. Chiou, J. H. Yen, C. L. Fang, H. L. Chen, and T. Y. Lin, "Authentication of medicinal herbs using PCR-amplified ITS2 with specific primers," Planta Medica, vol. 73, no. 13, pp. 1421-1426, 2007.

[26] W. J. Kress and D. L. Erickson, "A two-locus global DNA barcode for land plants: the coding rbcL gene complements the non-coding trnH-psbA spacer region," PloS ONE, vol. 2, no. 6, article e508, 2007. 
[27] R. Lahaye, M. van der Bank, D. Bogarin et al., "DNA barcoding the floras of biodiversity hotspots," Proceedings of the National Academy of Sciences of the United States of America, vol. 105, no. 8, pp. 2923-2928, 2008.

[28] C. P. Meyer and G. Paulay, "DNA barcoding: error rates based on comprehensive sampling," PLoS Biology, vol. 3, no. 12 article e422, 2005.

[29] R. Meier, K. Shiyang, G. Vaidya, and P. K. Ng, "DNA barcoding and taxonomy in Diptera: a tale of high intraspecific variability and low identification success," Systematic Biology, vol. 55, no. 5, pp. 715-728, 2006.

[30] A. Keller, F. Förster, T. Müller, T. Dandekar, J. Schultz, and M. Wolf, "Including RNA secondary structures improves accuracy and robustness in reconstruction of phylogenetic trees," Biology Direct, vol. 5, article 4, 2010.

[31] H. A. Ross, S. Murugan, and W. L. S. Li, "Testing the reliability of genetic methods of species identification via simulation," Systematic Biology, vol. 57, no. 2, pp. 216-230, 2008.

[32] M. W. Chase, R. S. Cowan, P. M. Hollingsworth et al., "A proposal for a standardised protocol to barcode all land plants," Taxon, vol. 56, no. 2, pp. 295-299, 2007.

[33] M. A. Buchheim, A. Keller, C. Koetschan, F. Förster, B. Merget, and M. Wolf, "Internal transcribed spacer 2 (nu ITS2 rRNA) sequence-structure phylogenetics: towards an automated reconstruction of the green algal tree of life," PLOS ONE, vol. 6, no. 2, Article ID e16931, 2011.

[34] A. Grajales, C. Aguilar, and J. A. Sánchez, "Phylogenetic reconstruction using secondary structures of Internal Transcribed Spacer 2 (ITS2, rDNA): finding the molecular and morphological gap in Caribbean gorgonian corals," BMC Evolutionary Biology, vol. 7, article 90, 2007.

[35] S. M. Markert, T. Müller, C. Koetschan, T. Friedl, and M. Wolf, “'Y' Scenedesmus (Chlorophyta, Chlorophyceae): the internal transcribed spacer 2 rRNA secondary structure re-revisited," Plant Biology, vol. 14, no. 6, pp. 987-996, 2012.

[36] A. Mello, C. Napoli, C. Murat, E. Morin, G. Marceddu, and P. Bonfante, "ITS-1 versus ITS-2 pyrosequencing: a comparison of fungal populations in truffle grounds," Mycologia, vol. 103, no. 6, pp. 1184-1193, 2011.

[37] J. Song, L. Shi, D. Li et al., "Extensive pyrosequencing reveals frequent intra genomic variations of internal transcribed spacer regions of nuclear ribosomal DNA," PLoS ONE, vol. 7, no. 8, Article ID e43971, 2012.

[38] M. Hajibabaei, G. A. C. Singer, E. L. Clare, and P. D. N. Hebert, "Design and applicability of DNA arrays and DNA barcodes in biodiversity monitoring," BMC Biology, vol. 5, article 24, 2007.

[39] J. C. Engelmann, S. Rahmann, M. Wolf et al., "Modelling crosshybridization on phylogenetic DNA microarrays increases the detection power of closely related species," Molecular Ecology Resources, vol. 9, no. 1, pp. 83-93, 2009.

[40] P. Taberlet, E. Coissac, F. Pompanon et al., "Power and limitations of the chloroplast trnL (UAA) intron for plant DNA barcoding," Nucleic Acids Research, vol. 35, no. 3, article e14, 2007. 

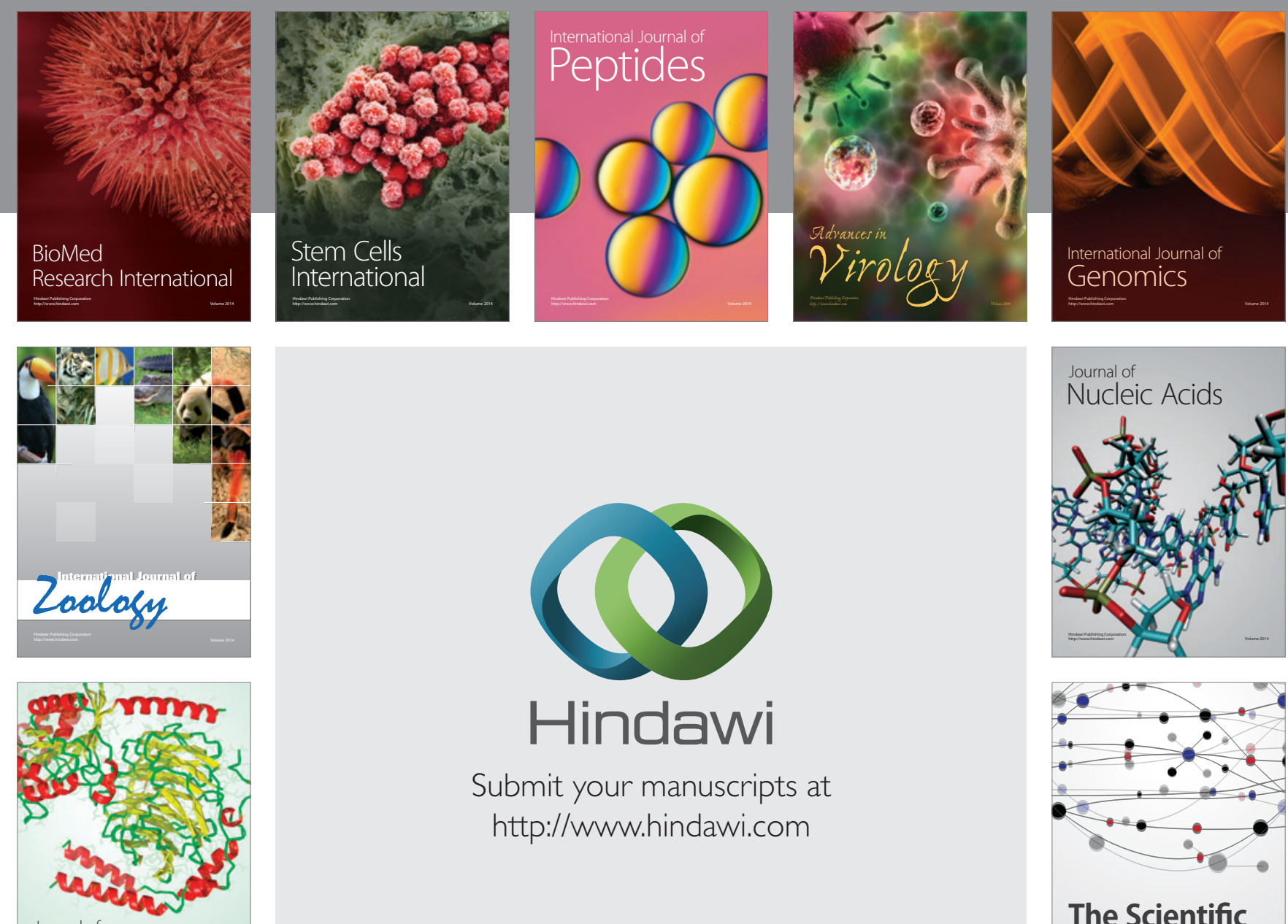

Submit your manuscripts at

http://www.hindawi.com

Journal of
Signal Transduction
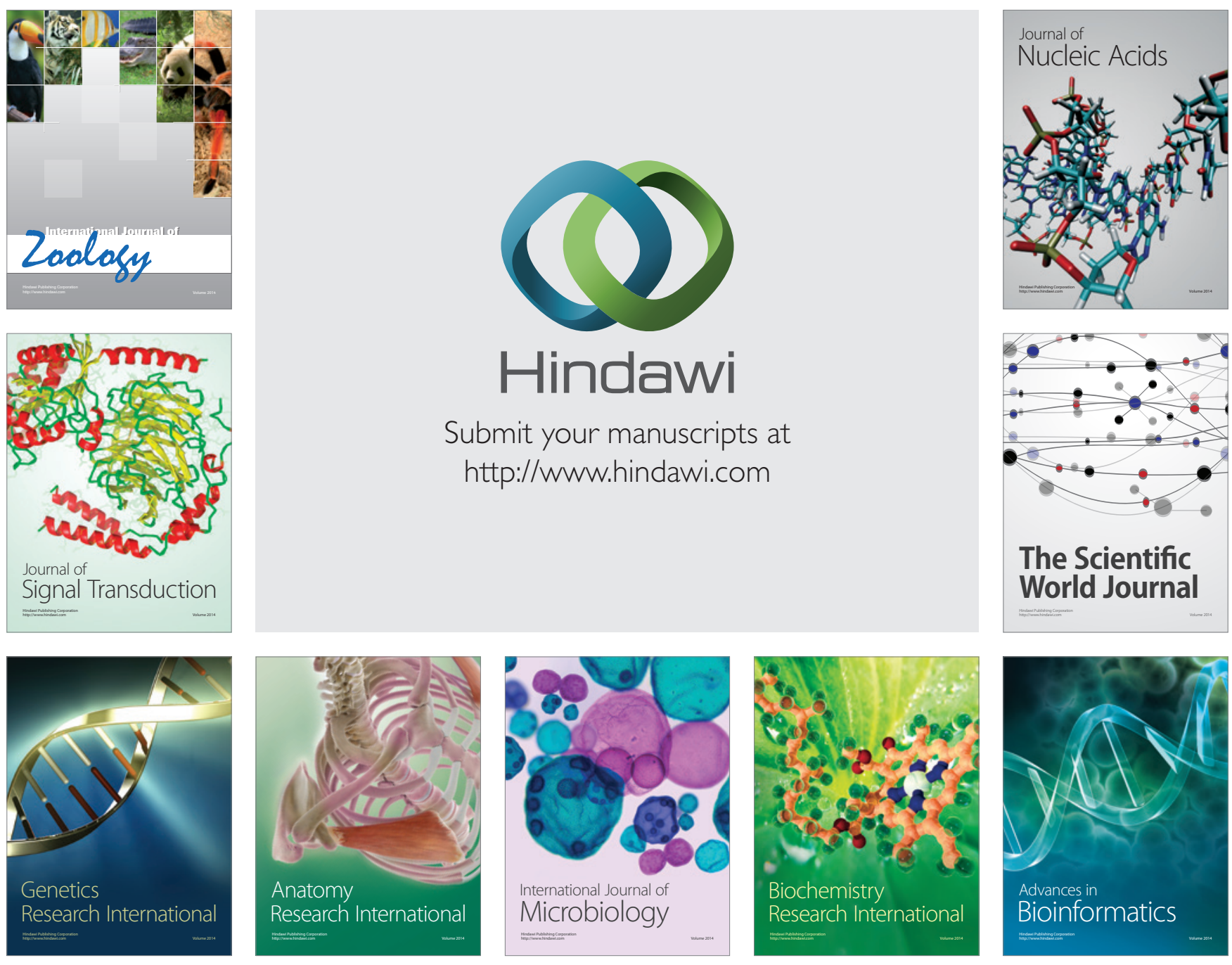

The Scientific World Journal
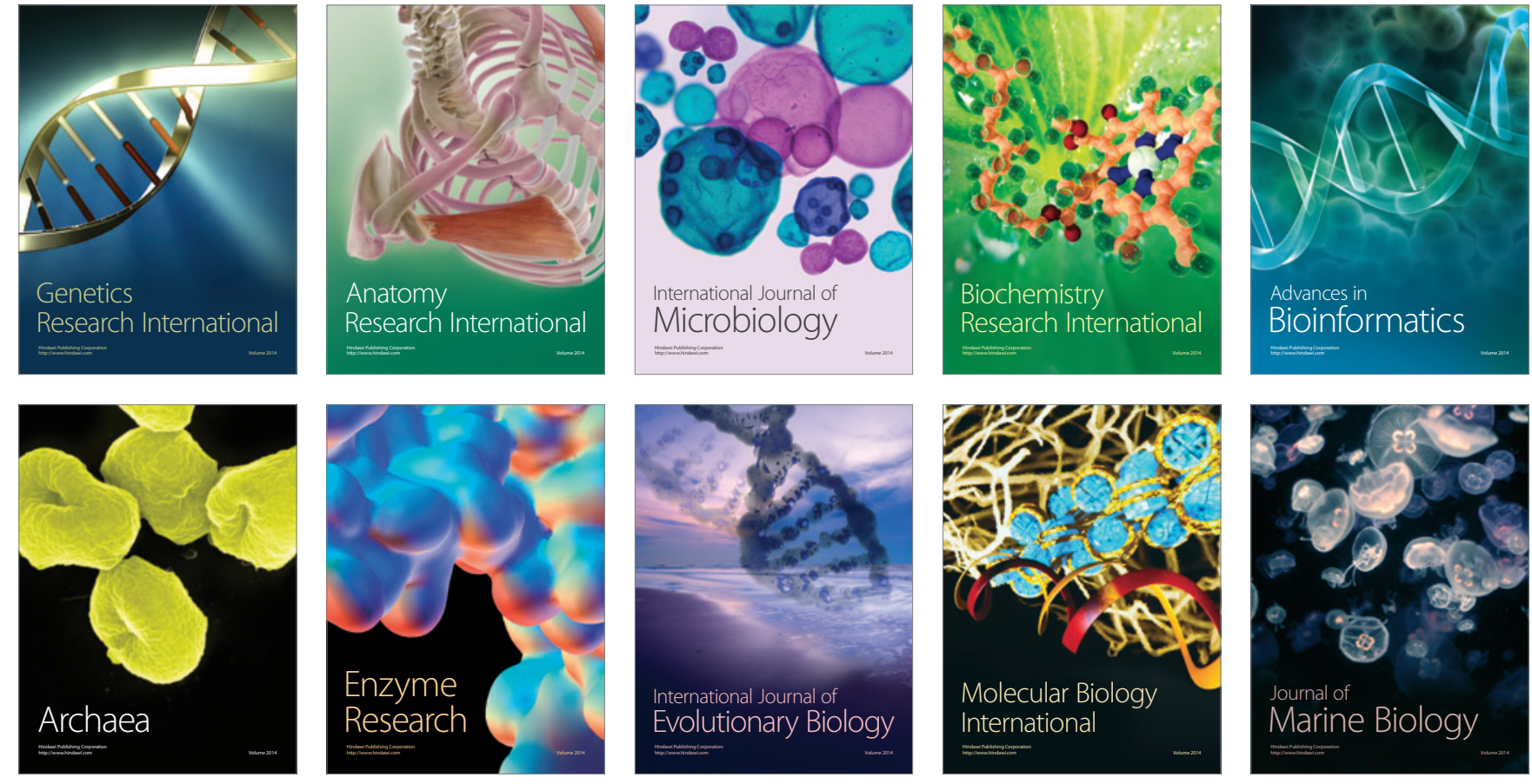